\title{
Human Placenta Extract Could Promote Proliferating Cell Nuclear Antigen Expression during Liver Regeneration Induced by Partial Hepatectomy in Rats
}

\author{
Ji Hyun Kim, Kyuboem $\mathrm{Han}^{1}$, Yong-Soo $\mathrm{Choi}^{2}$, Young Jun Lee ${ }^{1}$, Kwang Ho Yoon, \\ Man Deuk Han, Wan Jong Kim* \\ Department of Biology, Soonchunhyang University, Asan 336-745, Korea \\ ${ }^{1}$ CHA Bio \& Diostech Co., Ltd., Seoul 135-833, Korea \\ ${ }^{2}$ Department of Applied Bioscience, CHA University, Seongnam 463-836, Korea
}

*Correspondence to:

Kim WJ,

Tel: +82-41-530-1251

Fax: +82-41-530-1256

E-mail: wjkim56@sch.ac.kr

Received May 29, 2012

Revised September 8, 2012

Accepted September 9, 2012

\begin{abstract}
Human placenta extract (hPE) has therapeutic potential against certain diseases such as burn injury, liver cirrhosis and chronic wound through stimulating tissue repair processes. However, the effects of hPE on liver regeneration in animals are unknown. This study investigated the effect of hPE on the expression of proliferating cell nuclear antigen (PCNA) during liver regeneration induced by partial hepatectomy $(\mathrm{PH})$ in rats. The activities of AST, ALT and ALP increased during a few days after PH. A high level of ALP was particularly seen at day 3 in the control group. All the levels of experimental groups were normalized by day 5 after PH. On immunohistochemistry, the expression of PCNA increased at the early days, showed a peak at day 3 after PH. The PCNA staining was more obvious in the experimental group over the whole period. By western blotting, PCNA seemed to be more strongly expressed in the hPE injected group in the early stage and fell to almost undetectable levels at day 7. On immunocytochemical observations, the number of PCNA-gold particles in the nuclei at day 1 of the hPE treated groups was more than those of the untreated groups. The results suggest that hPE could accelerate liver regeneration induced by $\mathrm{PH}$ involving the expression of PCNA in rats.
\end{abstract}

Key Words: Human placenta extract, Liver regeneration, Partial hepatectomy, PCNA

\section{INTRODUCTION}

The liver has the unique capacity to regulate its growth and mass both in humans and in animals. In the partial hepatectomy $(\mathrm{PH})$ model, in which two thirds of the liver of a rat is removed, the residual liver lobes enlarge to compensate for the removed mass of the liver within 7 10 days (Higgins \& Anderson, 1931). This PH has been used as a surgical procedure for therapy of hepatitis, cirrhosis, liver transplantation and liver cancer. However, this procedure can lead to transient and even lethal liver failure in some patients.
Therefore, scientists are researching into ways to accelerate liver regeneration and decrease complications after $\mathrm{PH}$ (Fausto, 2004; Amemiya et al., 2011).

Liver regeneration is a highly complex and orchestrated process and its mechanism is not fully understood. Liver regeneration commenced immediately after $\mathrm{PH}$ is induced by specific external stimuli and involves sequential changes in gene expression, growth factor production, and morphological structure. Various cytokines (tumor necrosis factor [TNF], granulocyte colony-stimulating factor [G-CSF], macrophage CSF [M-CSF], granulocyte-macrophage CSF

@ This is an open-access article distributed under the terms of the Creative Commons Attribution Non-Commercial License (http://creativecommons.org/licenses/by-nc/3.0) which permits unrestricted noncommercial use, distribution, and reproduction in any medium, provided the original work is properly cited.

Copyrights (C) 2012 by Korean Society of Microscopy 
[GM-CSF]), growth factors (epidermal growth factor [EGF], hepatocyte growth factor [HGF], vascular EGF [VEGF], fibroblast growth factor [FGF]) and hormones (erythropoietin [EPO]) involved in the numerous steps of regeneration induced by $\mathrm{PH}$ increase the viability and accelerate liver repair through proliferation of hepatocytes and nonparenchymal cells (Assy \& Minuk, 1997; Gallucci et al., 2000; Fausto et al., 2006; Bockhorn et al., 2007).

Human placenta extract (hPE) is an extremely rich reservoir of bioactive molecules including hormones, cytokines, chemokines and growth factors such as HGF, EGF, FGF, transforming growth factor (TGF) and interleukin (IL) (Ansari et al., 1994). These factors participate in the process of cell interaction and signaling and lead to physiological actions of the biological system (Sur et al., 2003). hPE has been traditionally evaluated and used for treatment of psoriasis, rheumatoid arthritis, and others in Oriental medicine (Xue et al., 2003). Nowadays, hPE has been found to have therapeutic potential against certain diseases such as burn injury, chronic wound and has been used to treat a number of liver diseases including hepatitis and cirrhosis through stimulating tissue repair processes (Chakraborty \& Bhattacharyya, 2005). hPE immediately stimulated DNA synthesis of hepatocytes in vitro and influenced on the regulation of inflammatory response, stimulation liver regeneration and reduction of fibrosis in the CCl4-injured model (Wu et al., 2003).

Analysis of proliferating cell nuclear antigen (PCNA) protein has been used as an indication of cell proliferation. PCNA is an intranuclear protein of $36 \mathrm{kDa} \mathrm{MW}$, one of the cofactors of DNA polymerase $\delta$ (Assy et al., 1998).

In this study, we carried out a $\mathrm{PH}$ in rats and then investigated the in vivo effect of hPE on liver regeneration. Briefly, the ratio of liver regeneration, some enzyme activities and PCNA expression by western blotting, immunohistochemistry and immnogold labelling were determined and compared between the control and experimental groups.

\section{MATERIALS AND METHODS}

\section{Animals}

Male Sprague-Dawley rats, weighing 210 to $230 \mathrm{~g}$ (7 weeks old), were used. The animals were housed in air-conditioned specific pathegen free room at $21^{\circ} \mathrm{C}$ with a $12 \mathrm{hr}$ light/12 hr dark cycle and allowed food and water ad libitum. All experiments were performed following the Guideline for Animal Experimentation.

\section{Experimental Procedure}

Under ethyl ether anesthesia, 70\% PH involving the median and left lateral lobectomy was performed according to the procedure of Higgins and Anderson (1931). Rats were divided into 3 groups; normal group without PH (sham operated), control group injected with saline after $\mathrm{PH}$, and experimental group with subcutaneous injection of $100 \mu \mathrm{g} / 500 \mu \mathrm{L} \mathrm{hPE}$ (CHA Bio \& Diostech Co., Ltd., Seoul, Korea) every day from one day before $\mathrm{PH}$ to one day before sacrifice. Animals were sacrificed at 1, 3, 5, and 7 days after $\mathrm{PH}$. In the experiment, after the weights of the body and liver were recorded, blood and liver tissues of the rats were collected for blood chemistry analysis, immunohistochemical analysis, western blot analysis and immunocytochemical analysis.

\section{Calculation of Liver Weight and Recovery}

We calculated the ratio of liver regeneration at 1, 3, 5, and 7 days after $\mathrm{PH}$ according to the following equations (Mardrahimov et al., 2006);

Ratio of liver regeneration (\%)=regenerating remnant liver (g)/estimated whole liver (g)

Estimated whole liver $(\mathrm{g})=$ resected liver $\times 100 / 70$.

\section{Blood Chemistry Analysis}

Glutamic-oxalacetic-transaminase (GOT/AST), glutamic pyruvic-transaminase (GPT/ALT), and alkaline phosphatase (ALP) were measured in serum using an auto biochemistry detector.

\section{Immunohistochemical Analysis for PCNA}

Liver tissues obtained from sacrificed rats were fixed in $10 \%$ formalin and embedded in paraffin. Five- $\mu$ m thick sections were hydrated and washed with phosphate buffered saline (PBS). To block endogenous peroxidase activity, sections were injected with 3\% hydrogen peroxide diluted in PBS for $5 \mathrm{~min}$. Antibody nonspecific binding was blocked by incubating the sections for 15 min with $3 \%$ bovine serum albumin. Sections were incubated with primary antibody (1:300, PCNA mouse monoclonal antibody; Millipore, Darmstadt, Germany) overnight at $4^{\circ} \mathrm{C}$ and were washed 3 times with PBS. Samples were treated with biotinylated secondary antibody and incubated in streptavidin-HRP for $30 \mathrm{~min}$, respectively, and washed in PBS. Section were then stained with DAB (3,3'-diaminobenzidine). All steps were carried out at room temperature in a humidified chamber. Samples were examined by a light microscope (Jeong et al., 2010).

\section{Western Blot Analysis for PCNA}

Proteins of rat livers were lysed with protein extraction solution (PRO-PREPTM; Intron, Seongnam, Korea) and a homogenizer. Protein concentration was measured by the Lowry method. For sodium dodecyl sulfate polyacrylamide gel electrophoresis (SDS-PAGE), $40 \mu \mathrm{g}$ protein/lane was loaded onto a $10 \%$ sodium dodecyl sulfate polyacrylamide gel at $100 \mathrm{~V}$ for $90 \mathrm{~min}$. Protein was then transferred onto the hydrophobic polyvinylidene fluoride (PVDF) membrane (GE Healthcare, Seoul, Korea) at $300 \mathrm{~mA}$ for $40 \mathrm{~min}$. Blocking of 
membrane was carried out in 3\% skim milk for 30 min and then incubated with primary antibody (1:3,000, PCNA mouse monoclonal antibody) overnight at $4{ }^{\circ} \mathrm{C}$. Membrane was incubated with secondary antibody (1:3,000, goat anti-mouse IgG (H+L) HRP conjugate; Thermo Co., Somerset, NJ, USA). Protein signals were detected by enhanced chemiluminescence (ECL; Bionote, Hwaseong, Korea).

\section{Electron Microscopic Immunogold Labelling for PCNA}

Liver tissues were cut into pieces $\left(1 \mathrm{~mm}^{3}\right)$ and fixed in $4 \%$ glutaraldehyde in $0.1 \mathrm{M}$ phosphate buffer ( $\mathrm{PB}, \mathrm{pH}$ 7.4) for $4 \mathrm{hr}$. After rinsing in $\mathrm{PB}$, the samples were dehydrated with ethanol and embeded in araldite resin. The ultrathin sections were cut on an ultramicrotome and collected on nickel grids. The sections were incubated with saturated NaIO4. They were treated with 3\% BSA in EM-immunogold (EMG) buffer (0.05\% Tween, $0.5 \mathrm{M} \mathrm{NaCl}, 0.01 \mathrm{M} \mathrm{PB}, \mathrm{pH} 7.2)$ for $1 \mathrm{hr}$ each to block nonspecific reactions. They were then incubated in the presence of diluted monoclonal antibody to PCNA $(\times 100)$ at $4^{\circ} \mathrm{C}$ in a humidified chamber for $2 \mathrm{hr}$. The degree of non-specific labelling was checked in the present study by omission of primary antiserum (anti-PCNA). After rinsing in EMG buffer for 5 min 4 times, the ultrathin sections were incubated in $15 \mathrm{~nm}$ gold-conjugated goat anti-mouse IgG diluted $(\times 100)$ in EMG buffer for $2 \mathrm{hr}$, and then they were rinsed in PBS and distilled water. They were stained with only uranyl acetate. The labeled ultrathin sections were observed with a JEOL transmission electron microscope (JEOL, Tokyo, Japan). Additionally, the labeled gold particles were counted to compare their densities under the microscope (GonzálezMelendi et al., 2000; Ahn et al., 2005).

\section{RESULTS}

\section{Ratio of Liver Regeneration}

Liver regeneration was compared between the control groups and the experimental groups using previously regenerated/ generated equation. The wet weight of the remnant liver in the $70 \%$ PH group increased rapidly, reaching about $80 \%$ of the original liver weight at 5 days after surgery in both groups. However, at 7 days after surgery, the wet weight of the remnant liver of the hPE injected group reached nearly $100 \%$, compared with the untreated group of about $85 \%$. Full liver regeneration (100\% liver weight recovery) occurred at day 7 in the hPE injected rats. These results indicate the beneficial effect of $\mathrm{hPE}$ on liver regeneration induced by $\mathrm{PH}$ in rats (Fig. 1).

\section{Blood Chemistry Analysis}

Enzyme activities related with the liver function including GOT/AST, GPT/ALT and ALP were determined. In rats with $\mathrm{PH}$, the values for AST, ALT and ALP on the whole, increased in the early few days and normalized by day 5 in the hPE injected groups. However, the untreated groups were normalized by day 7 . ALP levels were significantly higher in the untreated animals by 3 days after operation compared with the hPE injected animals. hPE treatment might lead to a reduced liver damage after $70 \% \mathrm{PH}$ visible in terms of lower blood levels of liver enzymes. These data demonstrate that hPE could improve liver function of hepatic injured rats (Fig. 2).

Histology and Immunohistochemical Staining for PCNA Rat liver regeneration induced by $\mathrm{PH}$ was involved proliferation of cells including liver parenchymal and Kupffer cells. The disintegration and remodeling of hepatic plate, and increase of sinusoids were sequentially observed. The processes occurred earlier relatively in the experimental group (Fig. 3). To investigate whether hPE treatment could promote proliferation of hepatic cells in injured rats, we performed immunohistochemistry with PCNA antibody. The expression of PCNA, which is one of markers of cell proliferation, increased in the early stage, showed a peak at 3 days after $\mathrm{PH}$ and decreased thereafter. The expression of PCNA showed the peak at 1 day in the experimental groups. The levels at 7 days after $\mathrm{PH}$ were similar to baseline values. Comparatively, the intensities of PCNA staining were more obvious in the hPE injected group over the whole experimental period. These findings suggest that $\mathrm{hPE}$ administration on $\mathrm{PH}$ model could accelerate liver regeneration through improved proliferation activity of hepatocytes and nonparenchymal cells (Fig. 4).

\section{Western Blot Analysis}

The analysis of PCNA expression using western blotting appeared similar to that of immunohistochemical staining observation. PCNA was strongly expressed in the two groups in the early stage, and fell to almost undetectable levels at

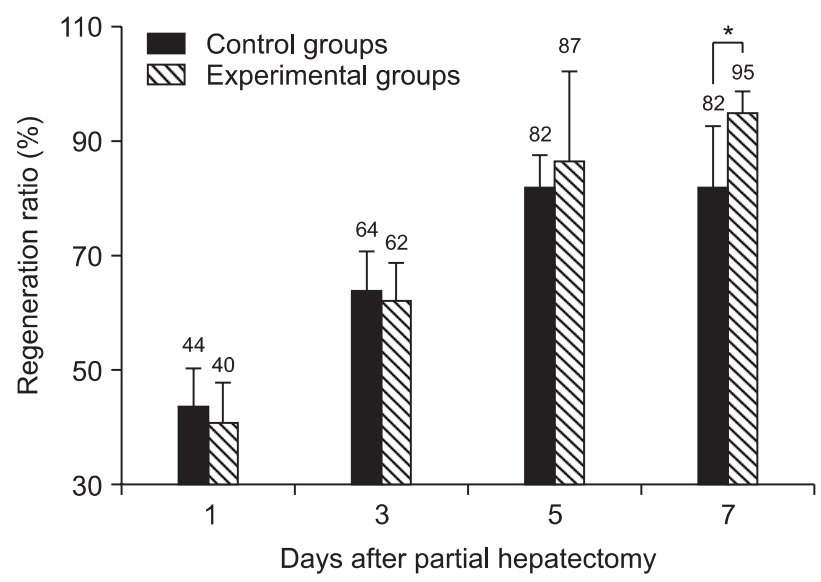

Fig. 1. Relative regeneration ratio in control and experimental groups at various days after partial hepatectomy. The results revealed significantly higher regeneration ratio in experimental group at 5 and 7 day. Each value and vertical bar represent the mean \pm standard deviation. ${ }^{*} \mathrm{p}<0.01$. 

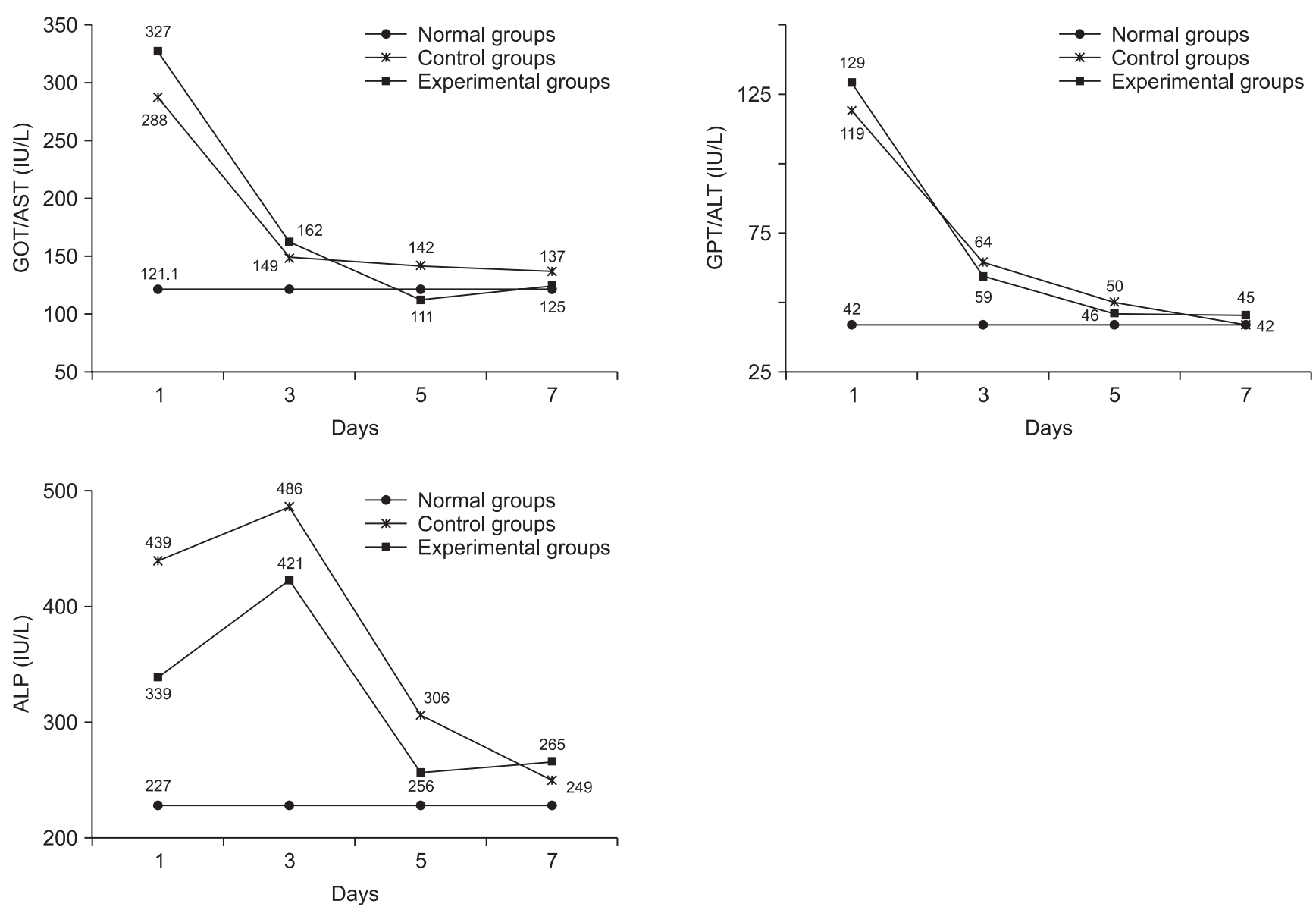

Fig. 2. Liver enzyme activities in the normal, control and experimental groups at various days after partial hepatectomy. Glutamic-oxalacetic-transaminase (GOT/AST), glutamic pyruvic-transaminase (GPT/ALT) and alkaline phosphatase (ALP) of experimental groups were similar to normal groups at 5 day.

7 days. In patricular, PCNA of the hPE injected groups were more strongly expressed compare to the untreated groups at day 1 . This suggests that hPE has a potential for the proliferation of hepatic cells and promotion of liver regeneration (Figs. 5 and 6).

\section{Immunocytochemical Analysis for PCNA}

Localization of PCNA proteins in hepatocytes and Kupffer cells were investigated by immunocytochemical observation using the gold labelling method. PCNA proteins were mostly localized in heterochromatin and nucleolus in the nucleus, and a few were in the cytoplasm of proliferating cells. Moreover, the number of PCNA-gold particles in Kupffer cells was higher than hepatocytes at 1 day. Number of intranuclear and extranuclear gold particles was randomly counted under an electron microscope of 20,000 magnifications. The result of nucleus/cytoplasm gold particles seemed to be in the ratio of eight to two. Also, the number of PCNA-gold particles in the nuclei at day 1 of the hPE groups was more than those of the untreated groups. Briefly, hPE immediately stimulated PCNA expression, thereby inducing DNA synthesis in heterochromatin and nucleolus and finally accelerating proliferation of hepatic cells (Figs. 7 and 8).

\section{DISCUSSION}

Liver regeneration is a result of highly coordinated proliferation of hepatocytes and non-parenchymal liver cells. This process is an orchestrated response induced by specific external stimuli involving sequential changes in gene expression, growth factor production, and morphological structure (Michalopoulos, 2007). PH is the most often used stimulus to study liver regeneration compared to other methods that use hepatic toxins because $\mathrm{PH}$ is not associated with the tissue injury and inflammation, and the initiation of the regenerative stimulus is precisely defined (Madrahimov et al., 2006).

$\mathrm{hPE}$, which is sometimes used to promote certain functions of liver or cure certain diseases such as hepatitis and liver cirrhosis through stimulating cell proliferation, has been known to promote IL-8 expression through in vitro and liver regeneration in the $\mathrm{CCl}_{4}$-injured liver rat model (Pal 
1 day
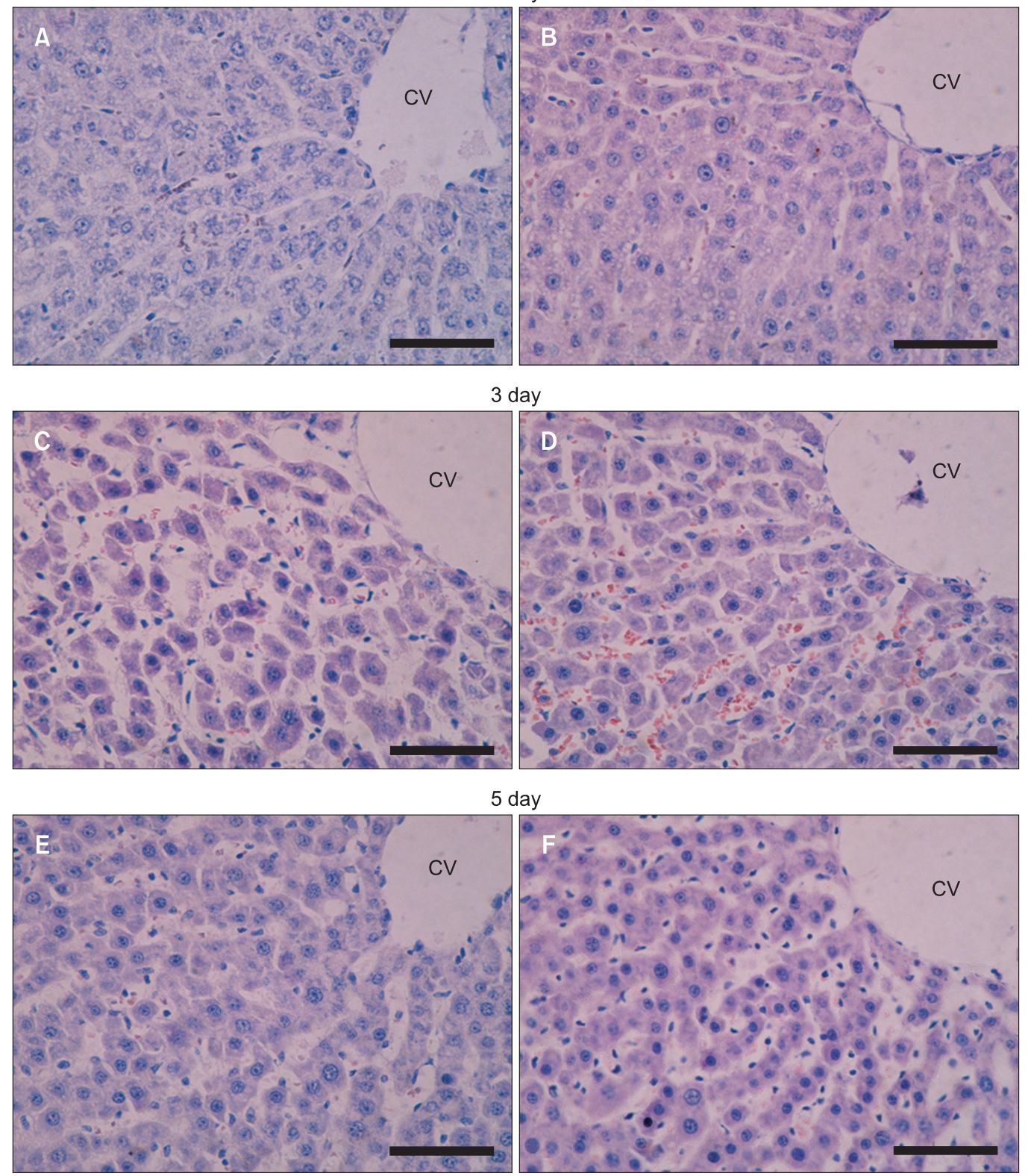

7 day

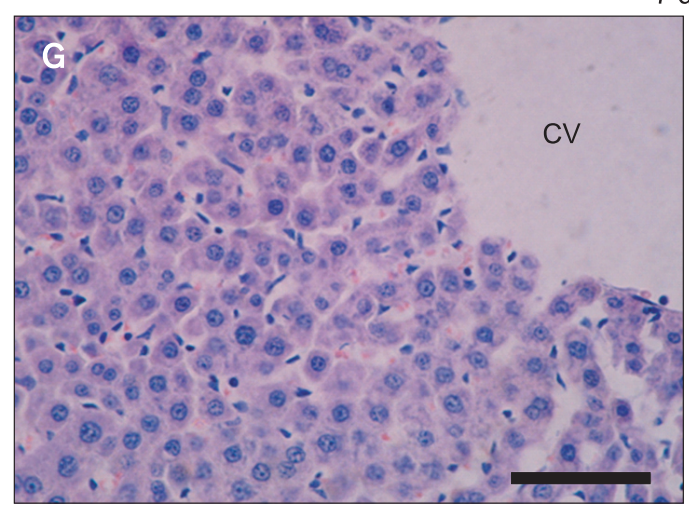

Control group

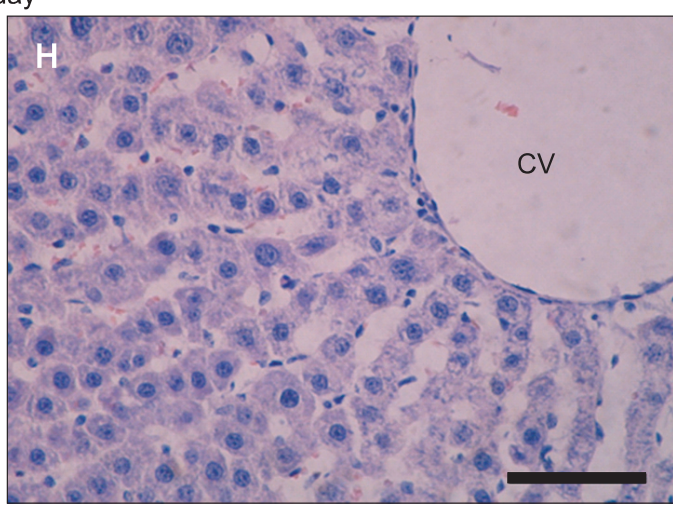

Experimental group

Fig. 3. Histology of the liver $1,3,5$, and 7 day after $70 \%$ partial hepatectomy (H\&E stain, Scale bar $=50 \mu \mathrm{m}$ ). CV, central vein. 

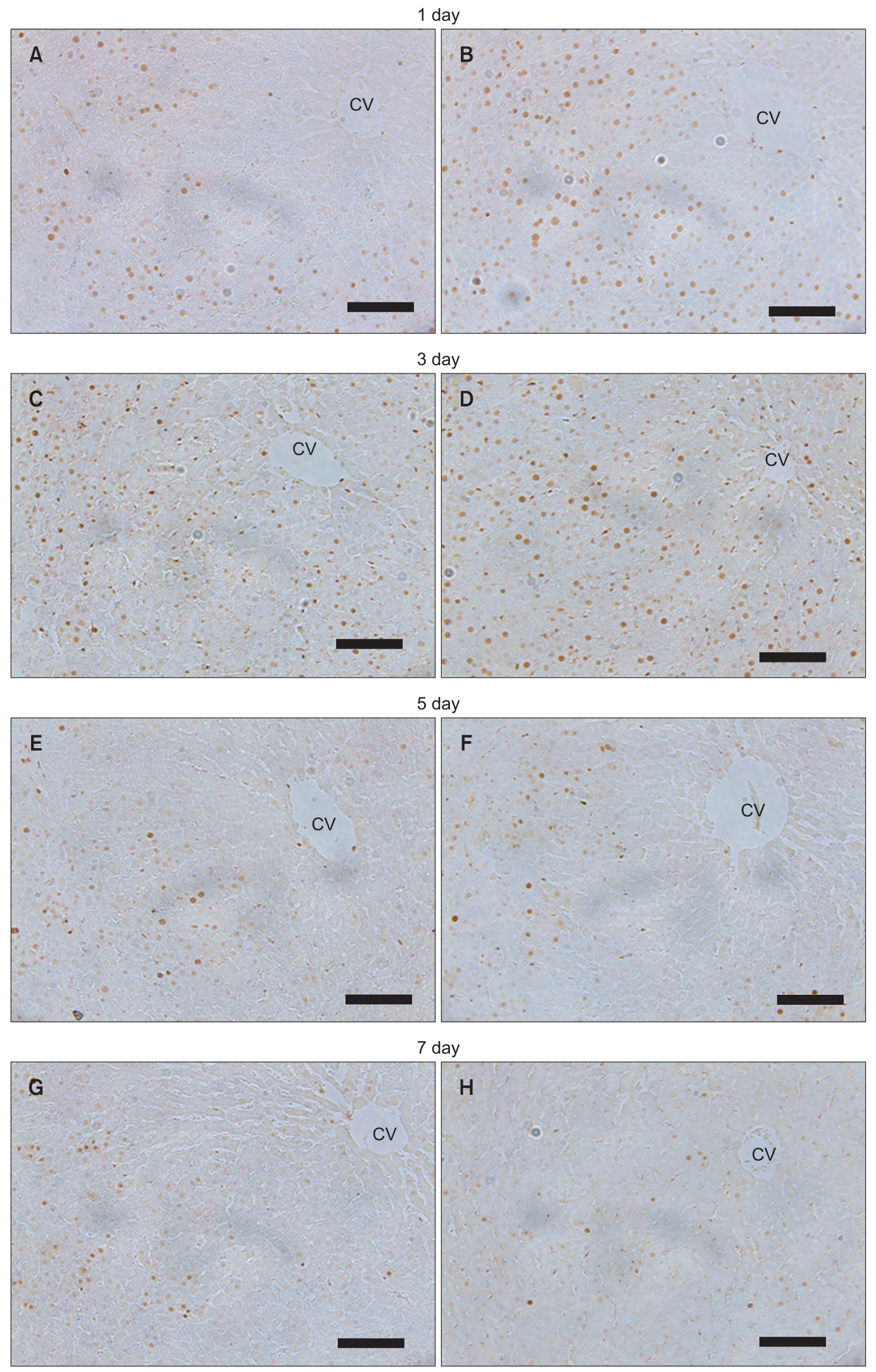

Control group

Experimental group

Fig. 4. Immunohistochemical staining for proliferating cell nuclear antigen of the liver at different time intervals following partial hepatectomy in rats. Scale bar $=100 \mu \mathrm{m}$. CV, central vein. 


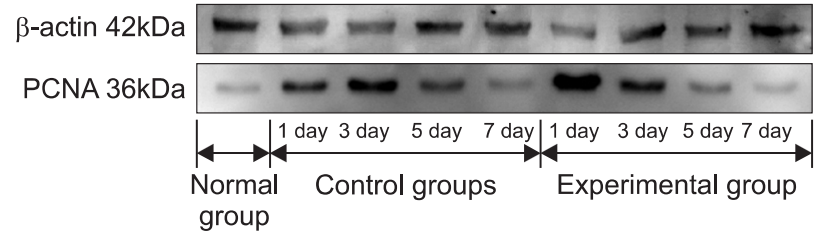

Fig. 5. Western blot of proliferating cell nuclear antigen (PCNA) expression in liver homogenates. Liver protein $(40 \mu \mathrm{g})$ was analysed by $10 \%$ sodium dodecyl sulfate polyacrylamide gel electrophoresis.

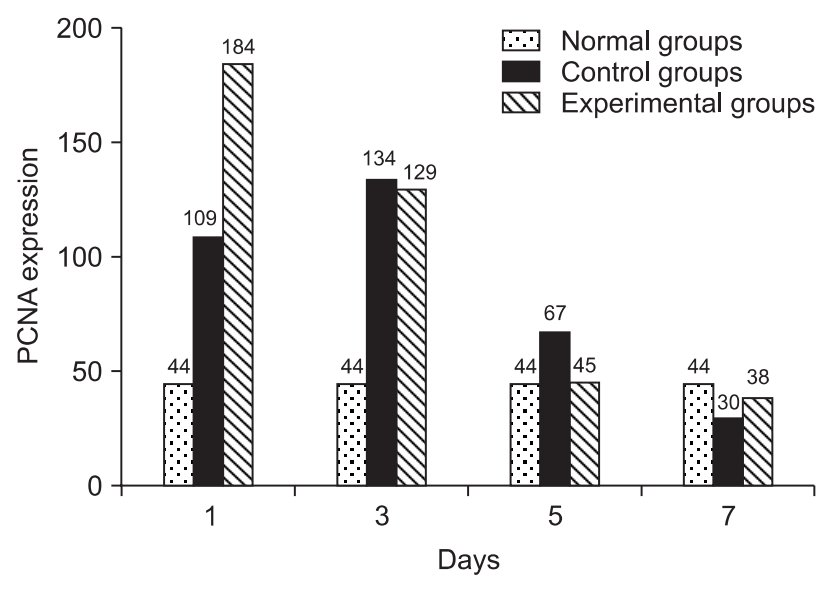

Fig. 6. Densitometric analysis of three separate samples in each group as in Fig. 5. Significant differences of proliferating cell nuclear antigen (PCNA) expression patterns were shown at 1 day and 5 day.

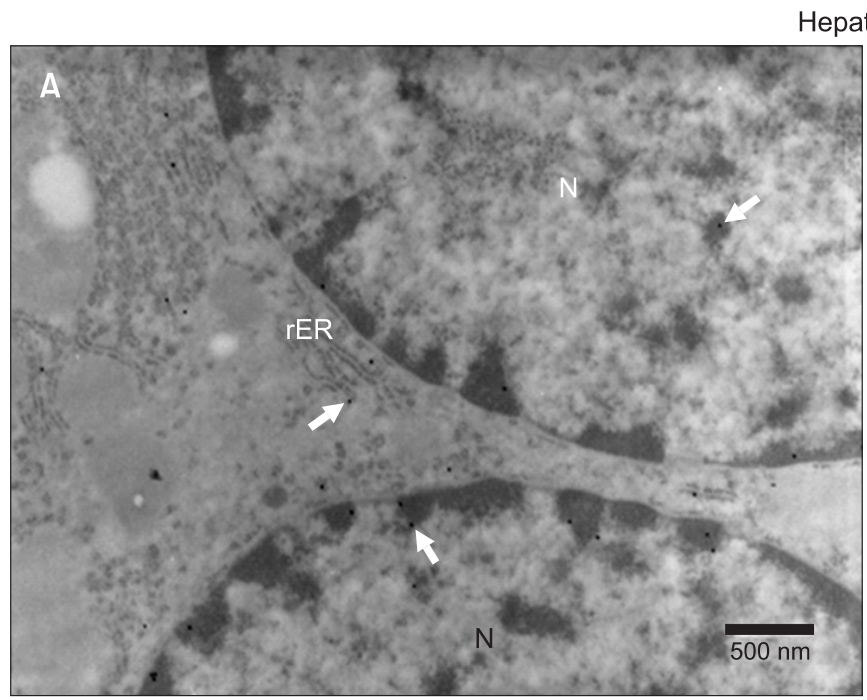

Control group

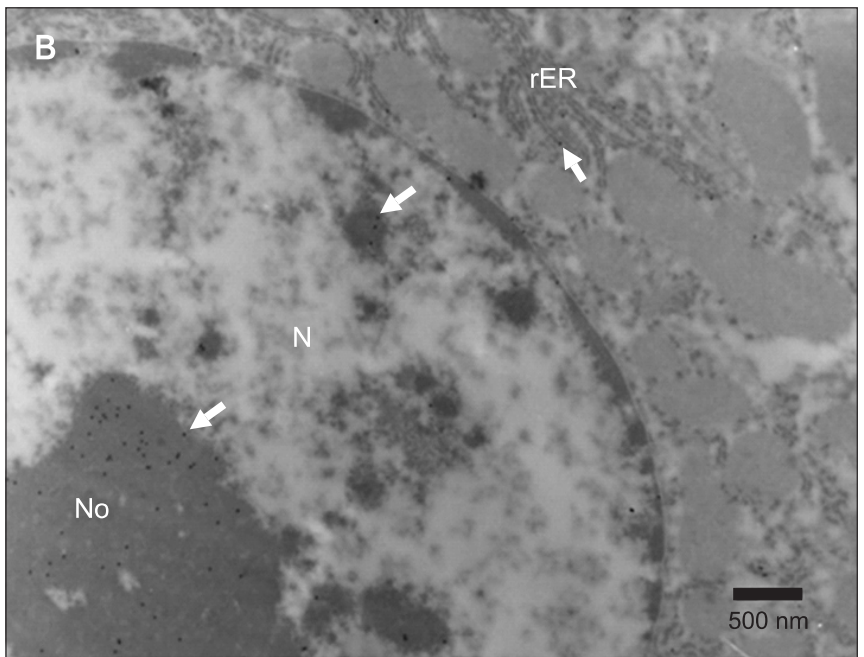

Experimental group

Kupffer cell

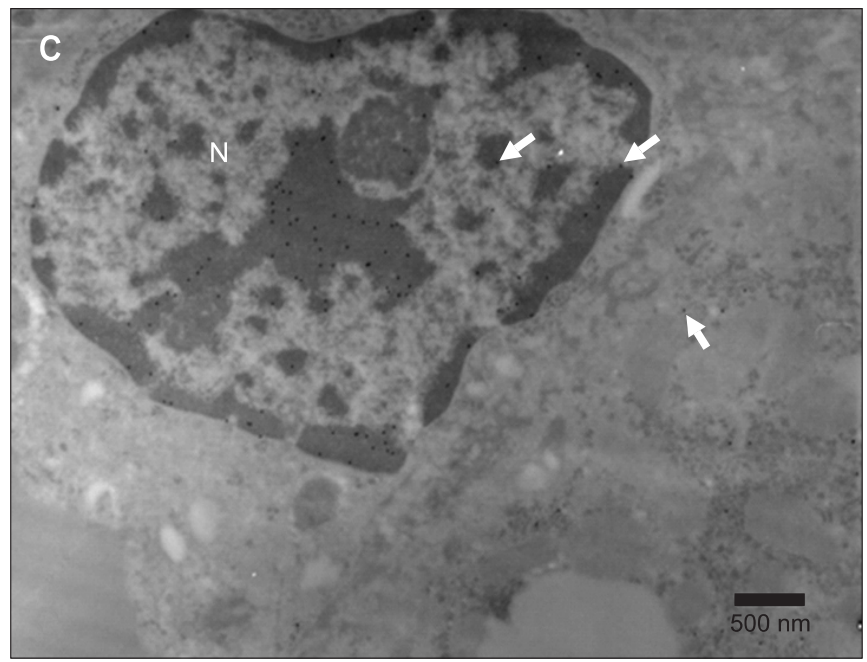

Control group

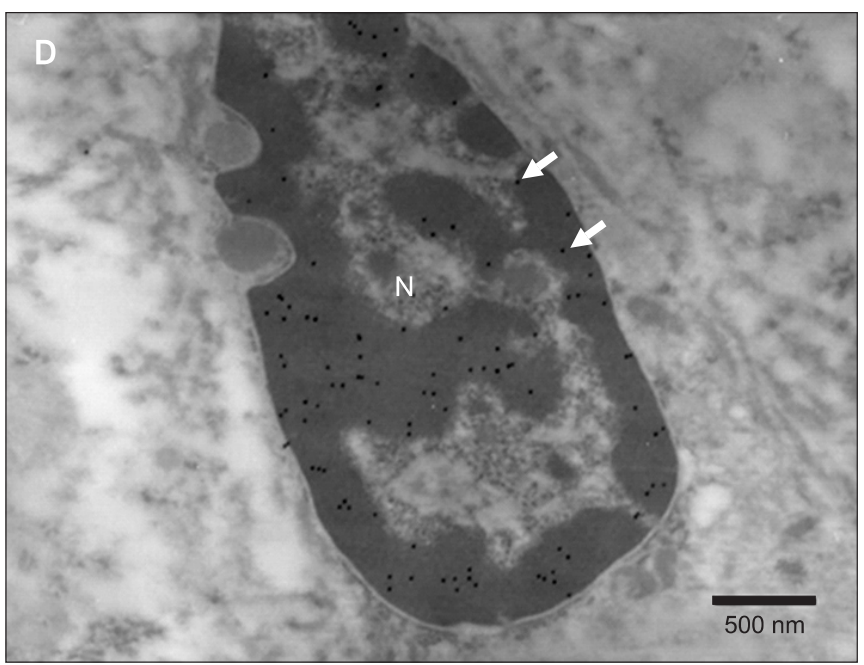

Experimental group

Fig. 7. Hepatocyte of normal group (A) and experimental group (B), Kupffer cell of control group (C) and experimental group (D) at 1 day. N, nucleus; No, nucleolus; rER, rough endoplasmic reticulum; arrows, proliferating cell nuclear antigen gold particles. 




Fig. 8. Number of intra, extranuclear and intracellular gold particles in cells per $\times 20,000$ viewing field at 1 day after partial hepatectomy. Each value and vertical bar represent the mean \pm standard deviation. ${ }^{*} \mathrm{p}<0.05$.

et al., 2002; Xue et al., 2003; Jung et al., 2011). PCNA, an intranuclear protein and a cofactor of DNA replication, has been used as an indicator of cell proliferation. In the present study, we demonstrated that hPE is capable of inducing $\mathrm{PCNA}$ in regenerating liver induced by $\mathrm{PH}$ in rats.

In hepatectomized rats, we observed a peak in hPE-induced PCNA expression. On immunohistochemical findings with the PCNA antibody, the expression of PCNA increased in the early stage, showed a peak at 3 days after $\mathrm{PH}$, and decreased thereafter. In particular, the expression of PCNA showed the peak at day 1 in the experimental groups. Comparatively, the intensities of PCNA staining were more obvious in the hPE injected group over the whole experimental period. These findings suggest that hPE administration on $\mathrm{PH}$ model could accelerate liver regeneration through improved proliferation activity of hepatocytes and nonparenchymal cells. Previously, we reported that PCNA was an indicator of cell proliferation and its level increased in the early liver regeneration in hepatectomized rats (Jeong et al., 2010). Our results showed a similar pattern in various methods for determination of PCNA. To localize the PCNA proteins in hepatocytes and Kupffer cells, we performed immunocytochemistry using the gold labelling method and observed under a tranasmission electron microscope. Numerous PCNA particles were clearly localized in chromatin and nucleolus in the nucleus. In the case of proliferating cells, a few particles were present in the cytoplasm. The number of PCNA-gold particles in Kupffer cells was more than hepatocytes at 1 day. This suggests that
Kupffer cells as well as hepatocytes were invovled in the process of liver regeneration after PH (Kelman, 1997; Takeishi et al., 1999). Meanwhile, the ratio of gold particles in the nucleus/cytoplasm seemed to be eight vs. two. In particular, the number of PCNA-gold particles in the nuclei at day 1 of the hPE groups was more than those of the untreated groups. Our results showed the similar tendency compared to previous reports (Liu et al., 1998; Tanno \& Taguchi, 1999; Jung et al., 2011).

In conclusion, the current study suggests that hPE immediately stimulates PCNA expression which indicates rapid DNA synthesis during liver regeneration of hPE injected rats.

\section{CONCLUSIONS}

$\mathrm{hPE}$, which is an extremely rich reservoir of bioactive molecules, has therapeutic potential against certain diseases such as burn injury, liver cirrhosis, inflammation, immune response and chronic wound through stimulating tissue repair processes. However, the effects of hPE on liver regeneration in animals including humans are still unknown. The present study investigated the in vivo effect of hPE on the expression of PCNA during liver regeneration induced by $\mathrm{PH}$ in rats. The ratio of regeneration was not comparatively different between the control and the experimental (hPE injected) groups. The activities of AST, ALT and ALP increased during a few days after PH. A high level of ALP was particularly seen at day 3 in the control group. All the levels of experimental groups were then normalized by day 5 after PH. On immunohistochemistry, the expression of PCNA increased at the early days, showed a peak at day 3 after $\mathrm{PH}$, and decreased thereafter. The PCNA staining was more obvious in the experimental group over the whole period. By western blotting, PCNA seemed to be more strongly expressed in the hPE injected group in the early stage and fell to almost undetectable levels at day 7. On immunocytochemical observations by the gold labelling method, the number of PCNA-gold particles in the nuclei at day 1 of the hPE treated groups was more than those of the untreated groups. The results suggest that hPE could accelerate liver regeneration induced by $\mathrm{PH}$ involving the expression of PCNA in rats. These findings are important for understanding the roles of $\mathrm{hPE}$ and provide evidence for therapeutic effects of hPE on hepatic injury. 


\section{REFERENCES}

Ahn Y M, Oh S H, Kim H J, Lee M Y, Lee J H, Shin K S, and Kim W J (2005) Immunogold labellings and expression of metallothionein in regenerating rat liver. Korean J. Electron. Microscopy 35, 15-22.

Amemiya $\mathrm{H}$, Kono $\mathrm{H}$, and Fujii $\mathrm{H}$ (2011) Liver regeneration is impaired in macrophage colony stimulating factor deficient mice after partial hepatectomy: the role of M-CSF-induced macrophages. J. Surg. Res. $165,59-67$.

Ansari K U, Nira G, and Bapat S K (1994) An experimental and clinical evaluation of immuno-modulating potential of human placental extract. Indian J. Pharmacol. 26, 130-132.

Assy N, Gong Y, Zhang M, Pettigrew N M, Pashniak D, and Minuk G Y (1998) Use of proliferating cell nuclear antigen as a marker of liver regeneration after partial hepatectomy in rats. J. Lab. Clin. Med. 131, 251-256.

Assy N and Minuk G Y (1997) Liver regeneration: methods for monitoring and their aplications. J. Hepatol. 26, 945-952.

Bockhorn M, Goralski M, Prokofiev D, Dammann P, Grunewald P, Trippler M, Biglarnia A, Kamler M, Niehues E M, Frilling A, Broelsch C E, and Schlaak J F (2007) VEGF is important for early liver regeneration after partial hepatectomy. J. Surg. Res. 138, 291-299.

Chakraborty P D and Bhattacharyya D (2005) In vitro growth inhibition of microbes by human placental extract. Current Science 88, 782-786.

Fausto N (2004) Liver regeneration and repair: hepatocytes, progenitor cells, and stem cells. Hepatology 39, 1477-1487.

Fausto N, Campbell J S, and Riehle K J (2006) Liver regeneration. Hepatol. 43, 45-53.

Gallucci R M, Simeonova P P, Toriumi W, and Luster M I (2000) TNFalpha regulates transforming growth factor-alpha expression in regenerating murine liver and isolated hepatocytes. J. Immunol. 164, 872-878.

Gonzalaz-Melendi P, Testollano P S, Ahmadian P, Reyes J, and Risueno M C (2000) Immunoelectron microscopy of PCNA as an efficient marker for studying replication times and sites during pollen development. Chromosoma 109, 397-409.

Higgins G M and Anderson R M (1931) Experimental pathology of the liver. Arch. Path. Lab. Med. 12, 186-202.
Jeong J J, Heo S H, Kim J H, Yoon K H, Lee Y J, Han K B, and Kim W J (2010) Effects of rrhGM-CSF in morphology and expression of PCNA in regenerating rat liver. Korean J. Microscopy 40, 73-80.

Jung J, Lee H J, Lee J M, Na K H, Hwang S G, and Kim G J (2011) Placenta extract promote liver regeneration in $\mathrm{CCl}(4)$-injured liver rat model. Int. Immunopharmacol. 11, 976-984.

Kelman Z (1997) PCNA: structure, functions and interactions. Oncogene 14, 629-640.

Liu K X, Kato Y, Kaku T, and Sugitama Y (1998) Human placental extract stimulates liver regeneration in rats. Biol. Pharm. Bull. 21, 44-49.

Madrahimov N, Dirsch O, Broelsch C, and Dahmen U (2006) Marginal hepatectomy in the rat: from anatomy to surgery. Ann. Surg. 244, 8998.

Michalopoulos G K (2007) Liver regeneration. J. Cell Physiol. 213, 286300.

Pal P, Mallick S, Mandal S K, Das M, Dutta A K, Datta P K, Bera R, and Bhadra R (2002) A human placental extract: in vivo and in vitro assessments of its melanocyte growth and pigment-inducing activities. Int. J. Dermatol. 41, 760-767.

Sur T K, Biswas T K, Ali L, and Mukherjee B (2003) Anti-inflammatory and anti-platelet aggregation activity of human placental extract. Acta. Pharmacol. Sin. 24, 187-192.

Takeishi T, Hirano K, Kobayashi T, Hasegawa G, Hatakeyama K, and Naito M (1999) The role of Kupffer cells in liver regeneration. Arch. Histol. Cytol. 62, 413-422.

Tanno M and Taguchi T (1999) Proliferating cell nuclear antigen in normal and regenerating rat liver. Exp. Mol. Pathol. 67, 192-200.

Wu C H, Chang G Y, Chang W C, Hsu C T, and Chen R S (2003) Wound healing effects of porcine placental extracts on rats with thermal injury. Br. J. Dermatol. 148, 236-245.

Xue F, Takahara T, Yata Y, Kuwabara Y, Shinno E, Nonome K, Minemura M, Takahara S, Li X, Yamato E, and Watanabe A (2003) Hepatocyte growth factor gene therapy accelerates regeneration in cirrhotic mouse livers after hepatectomy. Gut. 52, 694-700. 